\title{
Bit Error Rate Analysis of Cognitive Radio Network in the Presence of Primary Interference
}

\author{
Rajkumar Samikkannu
}

\begin{abstract}
In this paper, Bit Error Rate (BER) of Orthogonal Frequency Division Multiplexing (OFDM) based cognitive radio network is analyzed in the presence of Primary User Interference (PUI). Since, the PUI signal affects only few subcarriers of the OFDM signal, it can be modeled as a sparsity vector. In this network model, half duplex Amplify and Forward (AF) relaying is considered in the absence of direct link. Further, it is assumed that the network is operating in the overlay mode, thus the primary user transmitted signal act as interference at secondary user relay and destination nodes. In this paper, the PUI is mitigated using convex optimization. Bit Error Rate (BER) is analyzed using Quadrature Phase Shift Keying (QPSK) Modulation and Quadrature Amplitude Modulation (16-QAM). Simulation results conclude that after primary interference suppression, the performance of the proposed system is significantly improved.
\end{abstract}

Keywords: Cognitive Radio, OFDM, Amplify and Forward Relay and Primary User interference..

\section{INTRODUCTION}

Cognitive radio (CR) is a well-known technology developed to fulfill the demand of the available bandwidth [1]. CR systems operate in two modes such as the overlay mode and the underlay mode. In overlay mode, the secondary users occupy the band for the transmission when the primary users are absent. In underlay mode, the secondary user transmits the data over the band of primary users while the interference power from the secondary user falls below the threshold. Similarly, the primary transmitter signal acts as an interference to the nearby secondary receivers [2].

OFDM is another promising technology that supports the high data rate communications and it mitigates the multipath fading effect [3]. By combining with OFDM, the throughput of the CR network is highly improved. Further, to enhance the network coverage and the reliable transmission, the CR network uses the half duplex Amplify and Forward (AF) relaying strategy [4]. However, in practice, the proposed overlay cognitive radio network is inherently affected by the primary interference. In this paper, a compressive sensing algorithm is used to mitigate the PUI in OFDM based overlay cognitive relay network.

Recently, many of the research works are proposed in cognitive relay network with primary user interference [5]-[7]. The outage performance degradation due to the PUI in cognitive Decode and Forward (DF) relay network is analyzed in [5]. However, in this case, the mitigation of the

Revised Manuscript Received on 14 August, 2019.

Dr. Rajkumar Samikkannu, Professor, Department of ECE, Malla Reddy sraj.tce@gmail.com Engineering College for Women, Hyderabad-500100, India.

PUI is considered as a future work. In [6], the outage performance is analyzed in cognitive relay network using maximal ratio combining and selection combining with primary interference. Using sub-optimal power allocation, the outage probability of the cyclic prefixed single carrier spectrum sharing relay system with PUI is analyzed in [7]. Using Nakagami-m fading channel, the outage performance of the dual-hop DF relaying network is analyzed with the interference constraint in [8]. Degradation in outage due to the distance ratio of the interference link is discussed in cognitive relay networks and it achieves the full selection diversity as conventional diversity [9]. The outage performance is improved in underlay cognitive relay network with interference power constraint in [10].

Outage performance is the one of the performance metric to analyze any wireless system model and another important metric is the bit error probability. In [11], bit error performance of dual-hop DF relay network is analyzed over frequency flat fading. Bit Error Rate (BER) of the DF relay network is compared with optimal DF relay network using the optimal weight in [12]. Error probability of the multi-hop transmission is analyzed using the regenerative relays in [13]. Recently, the performance of the multi antennas AF relay network is analyzed with co-channel interference in [14].

In this paper, BER of the OFDM based cognitive relay network is analyzed and analytical expression is derived for primary user interference estimation. In earlier, interference mitigation is performed using filtering methods, cyclo-stationary concept and higher order statistics approach [15]. Recently, interference suppression is analyzed using compressive sensing algorithm, due to the sparsity nature of the interference in OFDM system [16].

The major contribution of this paper is that the suppression of primary user interference in cognitive relay network through the compressive sensing approach. Interference suppression of the proposed system is derived using convex optimization technique. Using the Minimum Mean Square Error (MMSE) estimation, data detection at the receiver is performed.

The rest of the paper contains the following sections. The system model of the proposed network is described in section II. In section III, the suppression of primary user interference is explained using the convex optimization technique. Numerical results are discussed in section IV. Finally, the conclusion is given in Section V.

Notations: Upper case and lower case letters with boldface $\boldsymbol{A}, \boldsymbol{a}$ indicates the matrix and vector respectively. Scalar is indicated by the lower case letter without boldface. 


\section{Bit Error Rate Analysis of Cognitive Radio Network in the Presence of Primary Interference}

\section{SYSTEM MODEL}

Consider a cognitive radio relay network with secondary source node $\mathrm{S}$, relay node $\mathrm{R}$ and destination node $\mathrm{D}$ as shown in Fig.1. It is assumed that primary user interference affects the secondary relay and destination nodes. Two time slots are needed for the half duplex transmission. In first time slot, source node transmits the signal to the relay and destination nodes and then in second slot, relay node transmits the signal to the destination node while the source node silent. In this model, half duplex transmission without direct link is considered.

The Channel Impulse Response (CIR) vector between the source node and relay node R is denoted by $\overline{\boldsymbol{h}}_{s r}$. Similarly, $\overline{\boldsymbol{h}}_{p r}$ is the CIR vector of primary user interference signal at relay node $\mathrm{R}$. The total length of the OFDM signal is $P=N+v$ which includes $N$ data subcarriers and $v$ zero-padded guard subcarriers. The guard sequence length, $v \geq L$ where $L$ is length of the channel taps which ensure the removal of Inter Symbol Interference (ISI).

The received vector $\overline{\boldsymbol{y}}_{R}$ at relay node $\mathrm{R}$ is expressed in time domain as

$$
\overline{\boldsymbol{y}}_{R}=\overline{\boldsymbol{H}}_{S R} \boldsymbol{F}_{2 p} \boldsymbol{x}_{S}+\overline{\boldsymbol{C}}_{\mathbf{l}} \overline{\boldsymbol{H}}_{P R} \overline{\boldsymbol{x}}_{P R}+\overline{\boldsymbol{n}}_{R}
$$

where $\boldsymbol{F}_{z p}=\left[\begin{array}{ll}\boldsymbol{F}_{N} & \boldsymbol{0}_{N \times \nu}\end{array}\right]^{H}$ is the $\boldsymbol{P} \times N$ Inverse Discrete Fourier Transform (IDFT) matrix, $\boldsymbol{x}_{S}$ is the $N \times 1$ input data sequence, $\boldsymbol{x}_{P R}=\boldsymbol{F} \overline{\boldsymbol{x}}_{P R}$ denote the frequency domain primary user interference signal which is in sparse, $\overline{\boldsymbol{H}}_{S R}$ and $\overline{\boldsymbol{H}}_{P R}$ are $P \times P$ time-domain circulant channel matrices, constructed by column-wise circularly shifting CIR vector $\overline{\boldsymbol{h}}_{x y}, \quad x y \in(S R, P R), \overline{\boldsymbol{n}}_{R}$ is the time domain complex Additive White Gaussian Noise (AWGN) vector. The elements of $\overline{\boldsymbol{n}}_{R}$ are independent and identically distributed with zero mean and variance $\sigma_{n}^{2}, \overline{\boldsymbol{C}}_{1}$ is the Carrier Frequency Offset (CFO) of the primary user signal $\overline{\boldsymbol{C}}_{1}=\operatorname{diag}[1, \exp (j 2 \pi \beta / P), \ldots, \exp (j 2 \pi \beta(P-1) / P)]$, where $\beta$ is random variable follow the uniform distribution.

By multiplying $\overline{\boldsymbol{y}}_{R}$ with DFT matrix $\boldsymbol{F}$, the received signal converted to frequency domain as given by

$$
\boldsymbol{y}_{R}=\boldsymbol{F} \overline{\boldsymbol{y}}_{R}=\boldsymbol{F}\left(\overline{\boldsymbol{H}}_{S R} \boldsymbol{F}_{z p} \boldsymbol{x}_{S}+\overline{\boldsymbol{C}}_{1} \overline{\boldsymbol{H}}_{P R} \overline{\boldsymbol{x}}_{P R}+\overline{\boldsymbol{n}}_{R}\right)
$$

Using Eigen Value Decomposition (EVD), the circulant matrices $\overline{\boldsymbol{H}}_{S R}$ and $\overline{\boldsymbol{H}}_{P R}$ are decomposed as $\overline{\boldsymbol{H}}_{S R}=\boldsymbol{F}^{H} \boldsymbol{\Lambda}_{S R} \boldsymbol{F}$, and $\overline{\boldsymbol{H}}_{P R}=\boldsymbol{F}^{H} \boldsymbol{\Lambda}_{P R} \boldsymbol{F}$. Thus, the signal $\boldsymbol{y}_{R}$ is simplified as

$$
\boldsymbol{y}_{R}=\boldsymbol{F} \bar{y}_{R}=\boldsymbol{\Lambda}_{S R} \boldsymbol{V} \boldsymbol{x}_{S}+\boldsymbol{C}_{1} \boldsymbol{\Lambda}_{P R} \boldsymbol{x}_{P R}+\boldsymbol{n}_{R}
$$

where $\boldsymbol{V}=\boldsymbol{F} \boldsymbol{F}_{z p}$ is the precoding matrix, $\Lambda_{S R}$ and $\Lambda_{P R}$ are the diagonal matrices whose elements are $P$-point DFT of the first column of $\overline{\boldsymbol{H}}_{S R}$ and $\overline{\boldsymbol{H}}_{P R}$ respectively. They are defined as $\lambda_{a b}=\left[\lambda_{a b}(0), \lambda_{a b}(1), \ldots, \lambda_{a b}(P-1)\right]$, where $a b \in(s r, p r)$ and $\boldsymbol{C}_{1}=\boldsymbol{F} \overline{\boldsymbol{C}}_{1} \boldsymbol{F}^{H}$ is the circulant CFO matrix, $\boldsymbol{n}_{R}=\boldsymbol{F} \bar{n}_{R}$ is the frequency domain noise signal.

In second time slot, relay node transmits the amplified signal to the destination node. The transmit signal at $k^{\text {th }}$ subcarrier is given as

$$
\begin{aligned}
x_{R}(k)= & \rho\left(\lambda_{S R}(k, k) v(k, k) x_{S}(k)\right. \\
& +\sum_{\substack{m=0 \\
m \in r}}^{P-1} \lambda_{P R}(k, m) c_{f o}\left(k,(m-k)_{P}\right) x_{P R}(m)+n_{R}(k)
\end{aligned}
$$

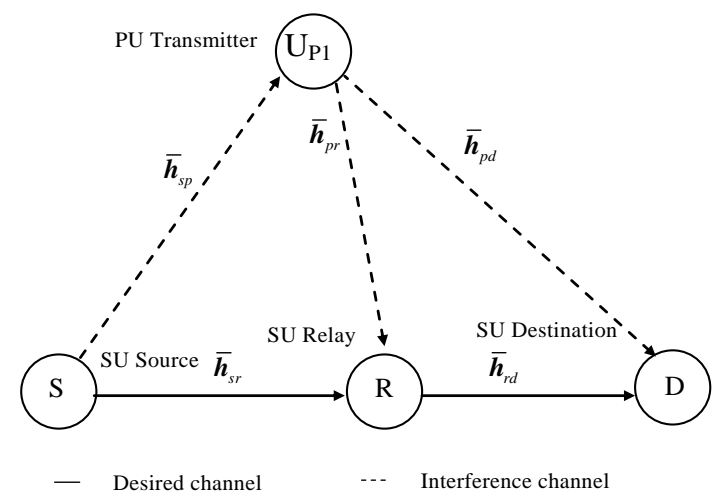

Fig. 1. Cognitive Amplify and Forward Relay Network.

where $\rho$ is the amplification factor, $r$ is the number of interference affected subcarriers. By taking expectation, (4) can be expressed as

$$
\begin{aligned}
& E\left(\left|x_{R}(k)\right|^{2}\right) \\
& =\rho\left(\left|\lambda_{S R}(k)\right|^{2}|v(k)|^{2} P_{s}+\sum_{m=0, m \in r}^{P-1}\left|\lambda_{P R}(m)\right|^{2} \varsigma_{R} \sigma_{P R}^{2}+\sigma_{n_{R}}^{2}\right)
\end{aligned}
$$

(5)

where $P_{s}=E\left(\left|x_{S}(k)\right|^{2}\right)$ is the source node transmit power, $\sigma_{n_{R}}^{2}=E\left(\left|n_{R}(k)\right|^{2}\right)$ is the variance of noise, the second term in (5) is achieved through Cauchy-Schwartz inequality

$$
\begin{gathered}
E\left(\left|\sum_{m=0, m \in r}^{P-1} \lambda_{P R}(m) c_{f o}\left(k,(m-k)_{P}\right) x_{P R}(m)\right|^{2}\right) \\
\leq \sum_{m=0, m \in r}^{P-1}\left|\lambda_{P R}(m)\right|^{2} \varsigma_{R} \sigma_{P R}^{2}
\end{gathered}
$$

where $\varsigma_{R}=\sum_{m=0, m \in r}^{P-1}\left|c_{f o}\left(k,(m-k)_{P}\right)\right|^{2}$ is the CFO squared norm, $\sigma_{P R}^{2}=E\left(\sum_{m=0, m \in r}^{P-1}\left|x_{P R}(m)\right|^{2}\right)$ is the Interference Power per Subcarrier (IPPS). Finally, the amplification factor can be chosen such that the relay node and source node transmit power are same.

$$
\rho(k) \geq \sqrt{\frac{P_{r}(k)}{\left|\lambda_{S R}(k)\right|^{2} P_{s}+\sum_{m=0, m \in r}^{P-1}\left|\lambda_{P R}(m)\right|^{2} \varsigma_{R} \sigma_{P R}^{2}+\sigma_{n_{R}}^{2}}}
$$


Since the CFO of primary interference signal affects the sparsity, it is necessary to apply the suitable window function to the received signal $\overline{\boldsymbol{y}}_{D}$ at destination node before taking the DFT. It reduces spectrum side lobes and improves the sparsity.

Apply the window function and then multiply with DFT matrix $\boldsymbol{F}$, received signal $\boldsymbol{y}_{D}$ is given by

$$
\boldsymbol{y}_{D}=\boldsymbol{F} \boldsymbol{\Lambda}_{W} \overline{\boldsymbol{y}}_{D}=\boldsymbol{F} \boldsymbol{\Lambda}_{W}\left(\overline{\boldsymbol{H}}_{R D} \overline{\boldsymbol{x}}_{R}+\overline{\boldsymbol{C}}_{2} \overline{\boldsymbol{H}}_{P D} \overline{\boldsymbol{x}}_{P D}+\overline{\boldsymbol{n}}_{D}\right)
$$

where $\Lambda_{W}=\operatorname{diag}[w(0), w(1), \ldots, w(P-1)]$ the window is function and $w(n)$ is the $n^{\text {th }}$ sample of window function. By substituting $\overline{\boldsymbol{x}}_{R}$ in (8), it can be written as

$$
\begin{aligned}
\boldsymbol{y}_{D}= & \rho \boldsymbol{H}_{1} \boldsymbol{\Lambda}_{R D} \boldsymbol{\Lambda}_{S R} \boldsymbol{V} \boldsymbol{x}_{S}+\rho \boldsymbol{H}_{2} \Lambda_{R D} \boldsymbol{C}_{1} \Lambda_{P R} \boldsymbol{x}_{P R} \\
& +\boldsymbol{H}_{3} \boldsymbol{C}_{2} \boldsymbol{\Lambda}_{P D} \boldsymbol{x}_{P D}+\boldsymbol{n}_{S R D}
\end{aligned}
$$

where $\boldsymbol{H}_{1}=\boldsymbol{F} \boldsymbol{\Lambda}_{W} \boldsymbol{F}^{H}, \boldsymbol{H}_{2}=\boldsymbol{F} \boldsymbol{\Lambda}_{W} \boldsymbol{F}^{H}$ and $\boldsymbol{H}_{3}=\boldsymbol{F} \boldsymbol{\Lambda}_{W} \overline{\boldsymbol{C}}_{2} \boldsymbol{F}^{H}$ are circulant matrices, $\tilde{\boldsymbol{n}}_{S R D}=\rho \boldsymbol{F} \overline{\boldsymbol{n}}_{R}+\boldsymbol{F} \boldsymbol{\Lambda}_{W} \overline{\boldsymbol{n}}_{D}$ is the frequency domain noise signal, $\Lambda_{R D}$ and $\Lambda_{P D}$ are the diagonal matrices whose elements are defined as $\lambda_{c d}=\left[\lambda_{c d}(0), \lambda_{c d}(1), \ldots, \lambda_{c d}(P-1)\right], \quad c d \in(r d, p d) . \quad$ As window function diminishes off diagonal elements of the circulant matrix, the second and third terms of (9) are well approximated as a sparse vector $\tilde{\boldsymbol{x}}_{P R} \square \rho \boldsymbol{H}_{2} \Lambda_{R D} \boldsymbol{C}_{1} \boldsymbol{\Lambda}_{P R} \boldsymbol{x}_{P R}$, $\tilde{\boldsymbol{x}}_{P D} \square \boldsymbol{H}_{3} \boldsymbol{C}_{2} \boldsymbol{\Lambda}_{P D} \boldsymbol{x}_{P D}$ which are the primary interference signals at relay and destination respectively, and $\tilde{\Lambda}_{S R D} \square \rho \boldsymbol{H}_{1} \Lambda_{R D} \Lambda_{S R} V$. Consequently, (9) can be simplified as

$$
\boldsymbol{y}_{D}=\tilde{\boldsymbol{\Lambda}}_{S R D} \boldsymbol{x}_{S}+\tilde{\boldsymbol{x}}_{P R}+\tilde{\boldsymbol{x}}_{P D}+\tilde{\boldsymbol{n}}_{S R D}
$$

Since, $\tilde{\boldsymbol{x}}_{P R}$ and $\tilde{\boldsymbol{x}}_{P D}$ are sparse vectors, it can be approximated as, $\tilde{\boldsymbol{x}}_{P R D} \approx \tilde{\boldsymbol{x}}_{P R}+\tilde{\boldsymbol{x}}_{P D}$ then (10) is written as

$$
\boldsymbol{y}_{D}=\tilde{\boldsymbol{\Lambda}}_{S R D} \boldsymbol{x}_{S}+\tilde{\boldsymbol{x}}_{P R D}+\tilde{\boldsymbol{n}}_{S R D}
$$

\section{Interference Mitigation Using Convex Optimization}

In this section, primary user interference estimation using compressive sensing algorithm is discussed. This well-known method is used to estimate the sparse like vectors from noisy under determined system. Convex optimization is familiar in signal processing and communication industry to solve the second order cone program. In this work, primary interference is estimated using convex optimization technique.

Since the signal and noise signals are unknown random vectors, first the signal term is blocked before the estimation of primary interference which reduces the estimation error. With the knowledge of Channel Frequency Response (CFR) vectors $\lambda_{s r}$ and $\lambda_{r d}$, the measurement matrix $\boldsymbol{M}$ is designed as a projection matrix which lie on the left null space of $\tilde{\Lambda}_{S R D}$.

$$
\boldsymbol{M}=\mathbf{I}_{P}-\tilde{\Lambda}_{S R D} \tilde{\Lambda}_{S R D}^{\dagger}
$$

where $\tilde{\Lambda}_{S R D}^{\dagger} \square\left(\tilde{\Lambda}_{S R D}^{H} \tilde{\Lambda}_{S R D}\right)^{-1} \tilde{\Lambda}_{S R D}^{H}$ is the Moore - Penrose pseudo inverse matrix.

By multiplying $\boldsymbol{y}_{D}$ by $\boldsymbol{M}$, the signal term is removed from the received signals and the resulting received signal $\hat{\boldsymbol{y}}_{D}$ contains only NBI and noise term

$$
\hat{\boldsymbol{y}}_{D}=\boldsymbol{M} \boldsymbol{y}_{D}=\boldsymbol{M} \tilde{\boldsymbol{x}}_{P R D}+\hat{\boldsymbol{n}}_{S R D}
$$

where $\hat{\boldsymbol{n}}_{S R D}=\boldsymbol{M} \tilde{\boldsymbol{n}}_{S R D}$ is the noise term. Now, (13) becomes the under determined system and able to apply the convex optimization technique to estimate the primary user interference. The estimation contains two steps such as problem formulation and interference parameter estimation. The problem is formulated using the following objective function and constraints

$$
\begin{aligned}
& \text { minimize }\left\|\tilde{\boldsymbol{x}}_{P R D_{\mathrm{eqv}}}\right\|_{1} \\
& \text { subject to }\left\|\hat{\boldsymbol{y}}_{D}-\boldsymbol{M} \tilde{\boldsymbol{x}}_{P R D \mathrm{eqv}}\right\|_{2}^{2} \leq \boldsymbol{\varepsilon}
\end{aligned}
$$

where $\|x\|_{1}$ and $\|x\|_{2}$ are the unity norm and Euclidean norm respectively, and $\boldsymbol{\varepsilon}=\left\|\hat{\boldsymbol{n}}_{S R D}\right\|_{2}^{2}$ is the parameter used as an upper bound for the given constraint.

After estimation of $\tilde{\boldsymbol{x}}_{P R D_{\mathrm{eqv}}}$ using convex optimization, it can be cancelled from $\boldsymbol{y}_{D}$ and finally it can be written as

$$
\breve{\boldsymbol{y}}_{D}=\boldsymbol{y}_{D}-\tilde{\boldsymbol{x}}_{P R D_{\mathrm{eqv}}} \approx \tilde{\boldsymbol{\Lambda}}_{S R D} \boldsymbol{x}_{S}+\boldsymbol{n}_{S R D}
$$

The data detection from the interference suppressed received signal using MMSE estimation can be written as

$$
\hat{\boldsymbol{x}}_{S}=\varepsilon_{x} \boldsymbol{\Lambda}_{S R}^{H} \boldsymbol{\Lambda}_{R D}^{H} \mathbf{H}_{w}^{H}\left(\sigma_{n}^{2} I+\varepsilon_{x}^{2} \mathbf{H}_{W} \boldsymbol{\Lambda}_{R D} \boldsymbol{\Lambda}_{S R} \boldsymbol{\Lambda}_{S R}^{H} \boldsymbol{\Lambda}_{R D}^{H} \mathbf{H}_{w}^{H}\right)^{-1} \mathbf{y}_{D}
$$

where $\varepsilon_{x}=\left|x_{S}\right|^{2}$ is the signal power, since $\boldsymbol{\Lambda}_{S R}$ and $\boldsymbol{\Lambda}_{R D}$ are diagonal matrices, (16) can be rewritten as

$$
\hat{\boldsymbol{x}}_{S}=\varepsilon_{x} \boldsymbol{\Lambda}_{S R D}^{H} \mathbf{H}_{w}^{H}\left(\sigma_{n}^{2} I+\varepsilon_{x}^{2} \mathbf{H}_{W} \boldsymbol{\Lambda}_{S R D} \boldsymbol{\Lambda}_{S R D}^{H} \mathbf{H}_{w}^{H}\right)^{-1} \mathbf{y}_{D}
$$

\section{Numerical Results}

In this section, the BER performance of the OFDM based cognitive relay network is analyzed in the presence of PUI Modulation techniques such as Quadrature Phase Shift Keying (QPSK) and 16-Quadrature Amplitude Modulation (QAM) are considered for the simulation work. It is assumed that the number of interference affected subcarriers among 64 subcarriers is 3 and 8 . The parameters used in the simulations are listed in Table.1.

Table 1: Simulation Parameters

\begin{tabular}{|l|l|l|}
\hline \multirow{2}{*}{ S.No } & $\begin{array}{l}\text { Simulation parameters of the proposed } \\
\text { Cognitive Relay Network }\end{array}$ \\
\cline { 2 - 3 } & Parameters & Value \\
\hline 1. & OFDM subcarriers length, $N$ & 64 \\
\hline
\end{tabular}




\begin{tabular}{|l|l|l|}
\hline \multirow{2}{*}{ S.No } & $\begin{array}{l}\text { Simulation parameters of the proposed } \\
\text { Cognitive Relay Network }\end{array}$ \\
\cline { 2 - 3 } & Parameters & Value \\
\hline 2. & Guard length, $v$ & 20 \\
\hline 3. & $\begin{array}{l}\text { No of subcarriers affected with } \\
\text { PUI, } r\end{array}$ & 3 \\
\hline 5. & Carrier frequency offset, $\beta$ & 0.5 \\
\hline 6. & Modulation & $\begin{array}{l}\text { QPSK, } \\
\text { 16-QAM }\end{array}$ \\
\hline
\end{tabular}

Figure 2 shows the BER performance of the OFDM system operating with cognitive relay. The comb type OFDM symbol is used throughout the simulation in which training data inserted at every 16 subcarriers. The number of subcarriers in the OFDM symbol is 64, zero-padded guard sequence length is 20 and the number of interference affected subcarriers is 3. The QPSK modulation is used in this simulation. From this figure, it is observed that the BER performance of the proposed system becomes poor at high SNR due to the presence of the PUI. At $20 \mathrm{~dB}$ SNR, BER of the system with PUI is $1.6 \times 10^{-3}$ whereas the system after suppressing the PUI, it reduces to $8.4 \times 10^{-4}$. At the same SNR, the BER of the proposed system without interference is $2.6 \times 10^{-4}$. It is investigated that performance of the proposed system is significantly improved after compensation of PUI.

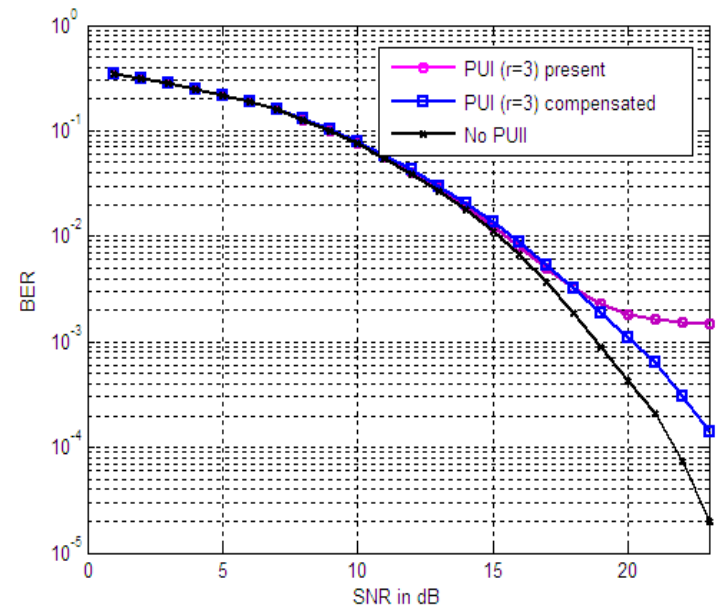

Fig. 2. BER performance of the proposed Cognitive AF relay network using QPSK modulation.

Figure 3 shows the BER performance of the proposed cognitive AF relay network using 16-QAM modulation. The number of subcarrier and interference affected subcarriers are same as in the previous figure. Figure shows that the performance of the proposed system after

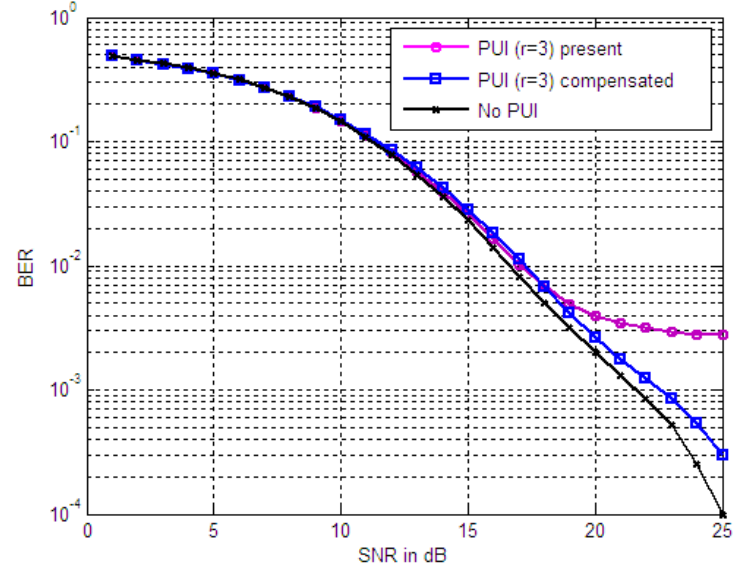

Fig. 3. BER performance of the proposed Cognitive network using 16-QAM modulation.

compensation of the PUI is approximately reaches to the network without PUI. It is observed that at $20 \mathrm{~dB}$ SNR, BER of the proposed cognitive relay network with PUI is $3.8 \times 10^{-3}$ whereas the network after suppressing PUI is $2.1 \times 10^{-3}$. Further, it is noted that the BER of the system without interference is $1.7 \times 10^{-3}$.

Figure 4 shows the BER performance of the OFDM based cognitive relay network. In this simulation eight subcarriers are considered as interference affected subcarriers. The number of subcarriers of the comb type OFDM symbol is 64 , and zero-padded guard sequence is 20. The modulation technique used in this simulation is QPSK. It is observed that the BER performance of the proposed cognitive relay network with 8 interference subcarriers is worse. However, the performance of the proposed network is significantly improved after interference suppression. At $20 \mathrm{~dB}$ SNR, error rate performance of the network with interference is 0.027 . For the same SNR, BER of the network is 0.0029 after suppressing the primary user interference. It concludes that in cognitive radio network, compressive sensing based interference estimation algorithm works well even though more interference are present.

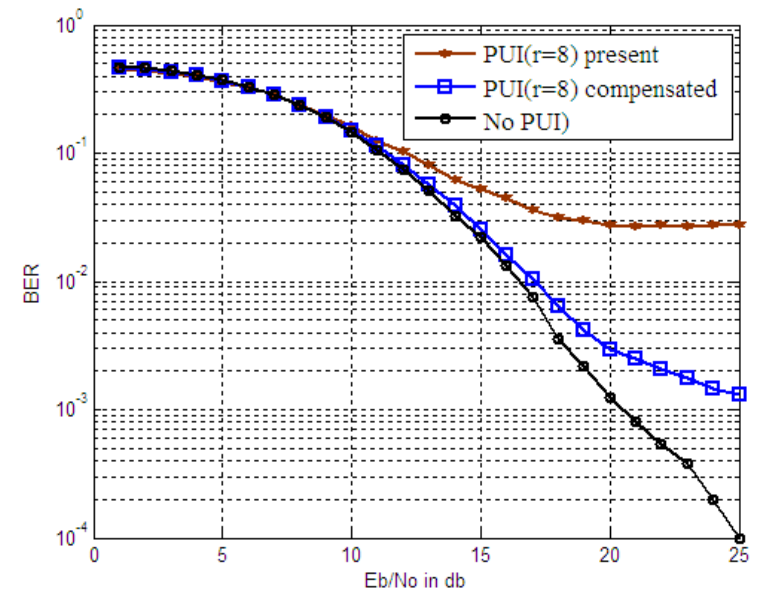

Fig. 4. BER performance of the proposed Cognitive network with 8 interference subcarriers using 16-QAM modulation.

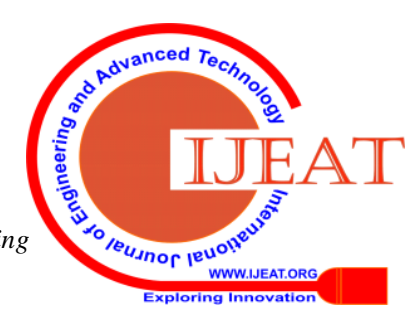


Figure 5 shows the comparison of the recovery of the interference signals with original interference signal. The number of subcarriers is 128 , and the interference affected subcarriers is 10 . The guard sequence length is chosen large, it is taken as 40 in order to exactly recover all the interference affected subcarriers. In this figure, small circles indicate the recovered subcarriers and star points indicate the desired and interference affected subcarriers of the transmitted OFDM symbol. At large guard sequence length, sparsity of the OFDM symbol is exploited and interference affected subcarriers are completely recovered.

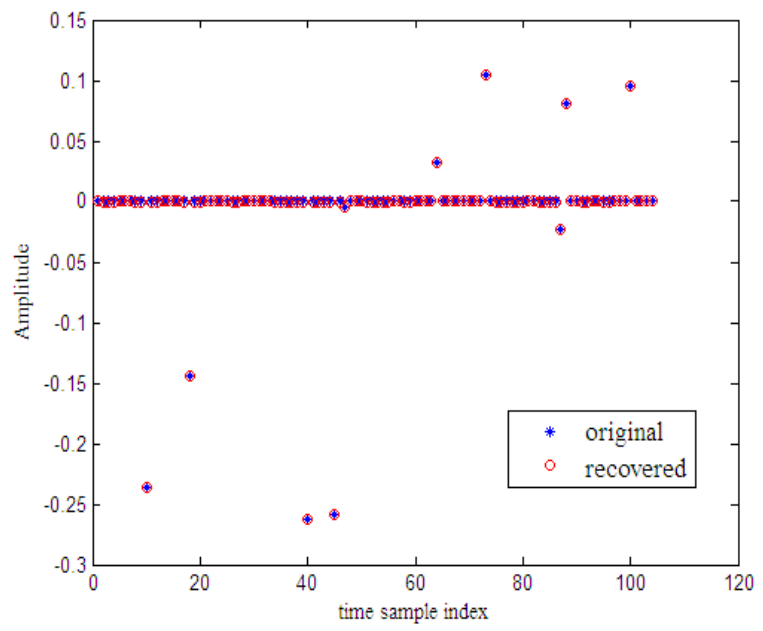

Fig. 5. Estimation of Primary User Interference in the proposed network using compressive sensing algorithm.

\section{CONCLUSION}

In this paper, BER of the OFDM based cognitive relay network is analyzed using QPSK and 16-QAM. Primary user interference signals at relay and destination nodes are modeled as a sparse vector. Using convex optimization technique, primary user interference is estimated. It is concluded that after mitigation of PUI, the performance of the proposed cognitive relay network is significantly improved. for your paper size.

\section{REFERENCES}

1. S. Haykin, "Cognitive radio: Brain-empowered wireless communications," IEEE J. Sel. Areas Commun., vol. 23, no.2, pp. 201-220, Feb. 2005.

2. Y. Zou, J. Zhu, B. Zheng, and Y. D. Yao, "An adaptive cooperation diversity scheme with best-relay selection in cognitive radio networks," IEEE Trans. Signal Process., vol. 58, no. 10, pp. 5438-5445, Oct. 2010

3. Bingham, J.A.C., (2008). "Multicarrier Modulation for Data Transmission: An Idea Whose Time Has Come," IEEE Commun Magazine, vol. 28, no.5, pp. 5-14.

4. J. N. Laneman, D. N. C. Tse and G. W. Wornell, “Cooperative diversity in wireless networks: efficient protocols and outage behavior," IEEE Trans. Inf. Theory, vol.50, no.12, pp. 3062-3080, Dec. 2004.

5. P. Yang, L. Luo and J. Qin, "Outage Performance of Cognitive Relay Networks with Interference from Primary User," IEEE Commun. Letters., vol. 10, no. 10, pp. 1695-1698, Oct. 2012.

6. W. Xu, J. Zhang, P. Zhang and C. Tellambura, "Outage Probability of Decode-and-Forward Cognitive Relay in Presence of Primary User's Interference ," IEEE Commun. Letters, vol. 16, no. 8, pp. 1252-1255, Aug. 2012.

7. K. J. Kim, T. Q. Duong, H. V. Poor and L. Shu, "Performance Analysis of Cyclic Prefixed Single-Carrier Spectrum Sharing Relay Systems in Primary User Interference,” IEEE Trans. Signal Process., vol. 60, no. 12, pp. 6729-6734, Dec. 2012.
8. C. Zhong, T. Ratnarajah, and K.K. Wong," Outage Analysis of Decode-and-Forward Cognitive Dual-Hop Systems With the Interference Constraint in Nakagami-mFading Channels," IEEE Trans. Veh. Technol., vol. 60, no.6, pp. 2875-2879, July 2011.

9. J. Lee, H. Wang, J. G. Andrews and D. Hong," Outage Probability of Cognitive Relay Networks with Interference Constraints,” IEEE Trans. Wireless Commun., vol. 10, no.2, pp. 390-395, Feb. 2011.

10. L. Luo, P. Zhang, G. Zhang and J. Qin," Outage Performance for Cognitive Relay Networks with Underlay Spectrum Sharing,'IEEE Commun. Letters, vol. 15, no.7, pp. 710-712, July 2011.

11. T.Satyanarayana, G.Upender, J.Surendiran , "Low Power Baseband Receiver Architecture Using STBC-OFDM With Flexible Mapper And Flexible Demapper For Mobile WMAN “, International Journal of Pure and Applied Mathematics Volume 119 No. 12 2018, 14337-14343 ISSN: 1314-3395.

12. G.A. Ropokis, A. A. Rontogiannis, and K. Berberidis, "BER Performance Analysis of Cooperative DaF Relay Networks and a New Optimal DaF Strategy ,'IEEE Trans. Wireless Commun., vol.10, no.4, pp. 1044-1049, Apr. 2011

a. Müller and J. Speidel,"Exact Symbol Error Probability of M-PSK for Multihop Transmission with Regenerative Relays," IEEE Commun. Letters, vol.11, no.12, pp. 952-954, Dec. 2007.

13. K.C. Lee, C. P. Li, T.Y. Wang and H.J. Li,"Performance Analysis of Dual-Hop Amplify and Forward Systems with Multiple Antennas and Cochannel Interference ,'IEEE Trans. Wireless Commun., vol.13, no.6, pp. 3070-3087, June 2014.

14. X. Hang, et.al,“ Cognitive Radio Networks: Interference Cancellation and Management Techniques,” IEEE Veh. Technol. Mag., vol. 4, no.4, pp. 76-84, Dec. 2009.

15. NagarjunaTelagam, S.Lakshmi , K.Nehru,“ BER analysis of concatenated levels of encoding in GFDM system using labview," Indonesian Journal of Electrical Engineering and Computer Science., vol.14,no.1,pp.8091,2019. DOI:10.11591/ijeecs.v14.i1

16. Rajesh, M., and J. M. Gnanasekar. "Path Observation Based Physical Routing Protocol for Wireless Ad Hoc Networks." Wireless Personal Communications 97.1 (2017): 1267-1289. 